\title{
Invariant random subgroups of semidirect products
}

\author{
Ian Biringer, Lewis Bowen ${ }^{\dagger}$ and Omer Tamuz
}

March 6, 2017

\begin{abstract}
We study invariant random subgroups (IRSs) of semidirect products $G=A \rtimes \Gamma$. In particular, we characterize all IRSs of parabolic subgroups of $\mathrm{SL}_{d}(\mathbb{R})$, and show that all ergodic IRSs of $\mathbb{R}^{d} \rtimes \mathrm{SL}_{d}(\mathbb{R})$ are either of the form $\mathbb{R}^{d} \rtimes K$ for some IRS of $\mathrm{SL}_{d}(\mathbb{R})$, or are induced from IRSs of $\Lambda \rtimes \operatorname{SL}(\Lambda)$, where $\Lambda<\mathbb{R}^{d}$ is a lattice.
\end{abstract}

\section{Contents}

1 Introduction 1

1.1 IRSs of special affine groups . . . . . . . . . . . . . . . . . . . . 2

1.2 IRSs of parabolic subgroups of $\mathrm{SL}_{d}(\mathbb{R}) \ldots \ldots \ldots \ldots$

1.3 Plan of the paper ....................... 5

2 IRSs in general semidirect products $\quad 5$

2.1 The cocycle $S_{H} \ldots \ldots \ldots \ldots \ldots$

2.2 Group actions preserving finite measures . . . . . . . . . . . . . 6

2.3 Transverse IRSs . . . . . . . . . . . . . . . . . . . . . . . . . . . . . . . . 7

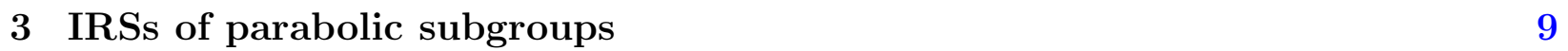

4 IRSs of special affine groups 12

\section{Introduction}

Let $G$ be a locally compact, second countable group and let $\mathrm{Sub}_{G}$ be the space of closed subgroups of $G$, considered with the Chabauty topology [9].

*Boston College. Supported in part by NSF grant DMS-1611851 and CAREER Award DMS-1654114.

${ }^{\dagger}$ University of Texas at Austin. Supported in part by NSF grant DMS-0968762, NSF CAREER Award DMS-0954606 and BSF grant 2008274.

${ }^{\ddagger}$ California Institute of Technology. This work was supported by a grant from the Simons Foundation (\#419427, Omer Tamuz). 
Definition 1. An invariant random subgroup (IRS) of $G$ is a random element of $\mathrm{Sub}_{G}$ whose law is a conjugation invariant Borel probability measure.

The term IRS was introduced by Abért-Glasner-Virág in [2], but the mathematical object has been studied earlier by Vershik [19]. Examples of IRSs include normal subgroups, as well as random conjugates $g \Gamma g^{-1}$ of a lattice $\Gamma<G$, where the conjugate is chosen by selecting $\Gamma g$ randomly against the given finite measure on $\Gamma \backslash G$. More generally, any IRS of a lattice $\Lambda<G$ induces an IRS of $G$ : if $\mu_{\Gamma}$ is the law of the original IRS and $\eta$ is a $G$-invariant probability measure on $\Gamma \backslash G$, the new law $\mu_{G}$ is given by the integral

$$
\mu_{G}=\int_{\Gamma g \in \Gamma \backslash G} g_{*} \mu_{\Gamma} d \eta
$$

where $\mu_{\Gamma}$ is regarded as a measure on $\mathrm{Sub}_{\Gamma} \subset \mathrm{Sub}_{G}$, and $g$ acts on $\mathrm{Sub}_{G}$ by conjugation. Informally, we conjugate the IRS of $\Gamma$ by an ' $\eta$-random' element of $G$. Since Sub $_{G}$ is compact [4, Lemma E.1.1], the space of (conjugation invariant) Borel probability measures on $\mathrm{Sub}_{G}$ is weak* compact, by Riesz's representation theorem and Alaoglu's theorem. Hence, IRSs compactify the set of lattices in $G$. There is a growing literature on IRSs (see, e.g., [3, 5, 6, $8,17])$ and their applications, see especially $[1,7,12,18]$.

Our goal in this note is to develop an understanding of IRSs of semidirect products $G=A \rtimes \Gamma$. There are few general constructions of such IRSs: there is the trivial IRS $\{e\}$, and IRSs of the form $A \rtimes K$, where $K$ is an IRS of $\Gamma$. When the kernel $\Gamma_{\text {triv }}$ of the action $\Gamma \circlearrowright A$ is nontrivial, one can also construct IRSs of the form $H \rtimes K$, where $H$ is an IRS of $A$ and $K$ is an IRS of $\Gamma$ that lies in $\Gamma_{\text {triv }}$, but additional examples are hard to find.

The kernel of our work are Theorems 2.5 and 2.6, in which we study 'transverse' IRSs of $G=A \rtimes \Gamma$ when $A$ is torsion-free abelian or simply connected nilpotent. Here, an IRS $H<G$ is transverse if $H \cap A=\{0\}$. This theorem has two parts: when $A$ is torsion-free abelian, we prove that that the projection of $H$ to $\Gamma$ acts trivially on $A$ almost surely, and if $A$ is a simply connected nilpotent Lie group, we show that an (often large) subgroup of $\Gamma$ acts precompactly on the Zariski closure of the set of all first coordinates of elements $(v, M) \in H$, as $H$ ranges through the support of the IRS.

As applications of Theorems 2.5 and 2.6, we study IRSs of two familiar semidirect products: the special affine groups $\mathbb{R}^{d} \rtimes \mathrm{SL}_{d}(\mathbb{R})$ and the parabolic subgroups of $\mathrm{SL}_{d}(\mathbb{R})$.

\subsection{IRSs of special affine groups}

We are particularly interested in IRSs of $\mathbb{R}^{d} \rtimes \mathrm{SL}_{d}(\mathbb{R})$. In addition to the examples $\{e\}$ and $\mathbb{R}^{d} \rtimes K$ mentioned above, one can construct an IRS from a lattice $\Lambda \subset \mathbb{R}^{d}$. Namely, the subgroup $\operatorname{SL}(\Lambda)<\mathrm{SL}_{d}(\mathbb{R})$ stabilizing $\Lambda$ is also a lattice, see [15], so the semidirect product $\Lambda \rtimes \mathrm{SL}(\Lambda)$ is a lattice in $\mathbb{R}^{d} \rtimes \mathrm{SL}_{d}(\mathbb{R})$, and hence a random conjugate of it is an IRS.

Theorem 1.1. Let $H$ be a non-trivial ergodic IRS of $\mathbb{R}^{d} \rtimes \mathrm{SL}_{d}(\mathbb{R})$. Then either

1. $H=\mathbb{R}^{d} \rtimes K$ for some $\operatorname{IRS} K<\mathrm{SL}_{d}(\mathbb{R})$, or 
2. $H$ is induced from an $\operatorname{IRS}$ of $\Lambda \rtimes \mathrm{SL}(\Lambda)$, for some lattice $\Lambda<\mathbb{R}^{d}$.

Here, an IRS is ergodic if its law is an ergodic measure for the conjugation action of $G$ on $\mathrm{Sub}_{G}$. By Choquet's theorem [14], every IRS can be written as an integral of ergodic IRSs. Note that by transitivity of the action of $\mathrm{SL}_{d}(\mathbb{R})$ on the space of lattices of a fixed covolume, we can actually choose $\Lambda$ in 2 . to be a scalar multiple of $\mathbb{Z}^{d}$.

As a corollary, any normal subgroup of $\mathbb{R}^{d} \rtimes \mathrm{SL}_{d}(\mathbb{R})$ is of the form $\mathbb{R}^{d} \rtimes K$ where $K$ is a normal subgroup of $\mathrm{SL}_{d}(\mathbb{R})$. (Here, $K=\{e\}, \mathrm{SL}_{d}(\mathbb{R})$ or $\{ \pm I\}$, where the last option is only available when $d$ is even.) Similarly, it follows that every lattice of $\mathbb{R}^{d} \rtimes \mathrm{SL}_{d}(\mathbb{R})$ is a finite index subgroup of some $\Lambda \rtimes \operatorname{SL}(\Lambda)$. We expect that these results are not entirely surprising, although we note that Theorem 4.8 of [10] is that $\mathbb{R}^{d} \rtimes \mathrm{SL}_{d}(\mathbb{R})$ has no uniform lattices, which follows trivially from this classification.

Stuck-Zimmer [16] show that for $d>2$, every ergodic IRS of $\mathrm{SL}_{d}(\mathbb{R})$ is either a lattice or a normal subgroup. This result, together with Theorem 1.1, implies that for $d>2$ every ergodic IRS of $\mathbb{R}^{d} \rtimes \mathrm{SL}_{d}(\mathbb{R})$ is likewise either a lattice or a normal subgroup.

In light of Theorem 1.1, to understand IRSs in special affine groups it suffices to study those of $G=\mathbb{Z}^{d} \rtimes \mathrm{SL}_{d}(\mathbb{Z})$. There are the usual examples $\{e\}$ and $\mathbb{Z}^{d} \rtimes K$, where $K$ is an IRS of $\mathrm{SL}_{d}(\mathbb{Z})$, but in general, some subtle finite group theory appears. For instance, let

$$
\pi_{n}: G \longrightarrow(\mathbb{Z} / n \mathbb{Z})^{d} \rtimes \mathrm{SL}_{d}(\mathbb{Z} / n \mathbb{Z})
$$

be the reduction map and setting $d=2$, consider the subgroup

$$
H=\left\{\left((t, 0),\left(\begin{array}{ll}
1 & 1 \\
0 & 1
\end{array}\right)^{t}\right) \mid t \in \mathbb{Z} / n \mathbb{Z}\right\}<(\mathbb{Z} / n \mathbb{Z})^{d} \rtimes \mathrm{SL}_{d}(\mathbb{Z} / n \mathbb{Z}) .
$$

The preimage $\pi_{n}^{-1}(H)$ is a finite index subgroup of $G$, and therefore can be considered as an IRS, but it does not have the form $\Lambda \rtimes K$ for any $\Lambda<\mathbb{Z}^{d}, K<\mathrm{SL}_{d}(\mathbb{Z})$. However, we will show that all IRSs of $G$ are semidirect products up to some 'finite index noise'. Namely, let

$$
G_{n}=\operatorname{Ker} \pi_{n}=n \mathbb{Z}^{d} \rtimes \Gamma(n),
$$

where $\Gamma(n)$ is the kernel of the reduction $\operatorname{map} \mathrm{SL}_{d}(\mathbb{Z}) \rightarrow \mathrm{SL}_{d}(\mathbb{Z} / n \mathbb{Z})$. We prove:

Theorem 1.2. Let $H$ be a non-trivial ergodic IRS of $\mathbb{Z}^{d} \rtimes \mathrm{SL}_{d}(\mathbb{Z})$. Then there is some $n \in \mathbb{N}$ such that $H_{n}=H \cap G_{n}$ is of the form $n \mathbb{Z}^{d} \rtimes K$, where $K$ is an IRS of $\mathrm{SL}_{d}(\mathbb{Z})$.

\subsection{IRSs of parabolic subgroups of $\mathrm{SL}_{d}(\mathbb{R})$}

Suppose that $W=\mathbb{R}^{d}$ is a finite dimensional real vector space, written as a direct sum

$$
W=S_{1} \oplus \cdots \oplus S_{n}
$$

of subspaces, and that $\mathcal{F}$ is the associated flag

$$
0=W_{0}<W_{1}<\cdots<W_{n}=W, \quad W_{k}=\oplus_{i=1}^{k} S_{i}
$$


Let $P<\mathrm{SL}(W)$ be the corresponding parabolic subgroup, i.e. the stabilizer of the flag $\mathcal{F}$, and let $V<P$ be the associated unipotent subgroup, consisting of all $A \in P$ that act trivially on each of the factors $W_{i} / W_{i-1}$. We then have

$$
P=V \rtimes R, \quad R=\left\{\left(A_{1}, \ldots, A_{n}\right) \in \prod_{i=1}^{n} \mathrm{GL}\left(S_{i}\right) \mid \prod_{i} \operatorname{det} A_{i}=1\right\} .
$$

Elements of $P$ can be considered as upper triangular $n \times n$-matrices, where the $i j^{\text {th }}$ entry is an element of $\mathcal{L}\left(S_{i}, S_{j}\right)$, the vector space of linear maps $S_{i} \longrightarrow S_{j}$. Elements of $R$ are diagonal matrices, and elements of $V$ are upper unitriangular.

Take a subset $\mathcal{E} \subset\{1, \ldots, n\}^{2}$ consisting of pairs $(i, j)$ with $i<j$ and such that if $(i, j) \in \mathcal{E}$, then $\left(i^{\prime}, j\right),\left(i, j^{\prime}\right) \in \mathcal{E}$ for $i^{\prime}<i$ and $j^{\prime}>j$. So, imagining elements of $\mathcal{E}$ as corresponding to matrix entries, we are considering subsets of entries above the diagonal, that are closed under 'going up' and 'going to the right'. Let $V_{\varepsilon}<P$ be the normal subgroup consisting of all matrices that are equal to the identity matrix except at entries corresponding to elements of $\mathcal{E}$, and let $\mathcal{K}_{\mathcal{E}}<R$ be the kernel of the $R$-action (by conjugation) on $V / V_{\mathcal{E}}$.

Theorem 1.3 (IRSs of parabolic subgroups). The ergodic IRSs of $P$ are exactly the random subgroups of the form $V_{\mathcal{\varepsilon}} \rtimes K$, where $K$ is an ergodic IRS of $\mathcal{K}_{\mathcal{\varepsilon}}$.

The subgroups $V_{\varepsilon}$ above are exactly the normal subgroups of $P$ that lie in $V$. So, a special case of the theorem is that an ergodic IRS of $P$ that is contained in $V$ is a normal subgroup of $P$. In fact, when proving Theorem 1.3, one first proves this special case, and then applies it to $H \cap V$ when $H$ is a general ergodic IRS of $P$. Once one knows $H \cap V=V_{\varepsilon}$, the statement of Theorem 1.3 is not a surprise, since the only obvious way to construct an IRS $H$ with $H \cap V=V_{\varepsilon}$ is to take a semidirect product with an IRS of $\mathcal{K}_{\varepsilon}$.

The group $\mathcal{K}_{\mathcal{E}}$ can be described explicitly via matrices. Let $\mathcal{J}$ be the set of all $i \in$ $\{1, \ldots, n\}$ such that if $i<n$, then $(i, i+1) \in \mathcal{E}$, and if $i>1$, then $(i-1, i) \in \mathcal{E}$. Then $\left(A_{1}, \ldots, A_{n}\right)$ acts trivially on $V / V_{\varepsilon}$ exactly when for each maximal interval $\{i, \ldots, j\} \subset$ $\{1, \ldots, n\} \backslash \mathcal{J}$, there is some $\lambda \in \mathbb{R} \backslash\{0\}$ such that $A_{i}=\cdots=A_{j}=\lambda I$. In a picture, if $\mathcal{E}$ consists of the starred entries below, then $\left(A_{1}, \ldots, A_{n}\right) \in \mathcal{K}_{\mathcal{E}}$ can be any diagonal matrix with the diagonal entries below, subject to the additional condition $\prod_{i} \operatorname{det} A_{i}=1$.

$$
\left(\begin{array}{cccccccc}
\lambda I & 0 & \star & \star & \star & \star & \star & \star \\
0 & \lambda I & \star & \star & \star & \star & \star & \star \\
0 & 0 & A_{3} & \star & \star & \star & \star & \star \\
0 & 0 & 0 & \mu I & 0 & 0 & \star & \star \\
0 & 0 & 0 & 0 & \mu I & 0 & \star & \star \\
0 & 0 & 0 & 0 & 0 & \mu I & \star & \star \\
0 & 0 & 0 & 0 & 0 & 0 & A_{7} & \star \\
0 & 0 & 0 & 0 & 0 & 0 & 0 & A_{8}
\end{array}\right)
$$

This means that $\mathcal{K}_{\varepsilon}$ is isomorphic to the quotient by a determinant condition of a direct product of general linear groups, some which are copies of $G L(\mathbb{R}) \cong \mathbb{R} \backslash\{0\}$. Note that the 
conjugation action of every element of $R$ on $\mathcal{K}_{\varepsilon}$ is equal to a conjugation by an element of $\mathcal{K}_{\varepsilon}$, since $R$ is generated by $\mathcal{K}_{\varepsilon}$ and its centralizer. So, every IRS of $\mathcal{K}_{\varepsilon}$ is an IRS of $R$.

\subsection{Plan of the paper}

The paper is organized as follows. In $\S 2$, we establish some preliminary results: we introduce in $\S 2.1$ a useful co-cycle associated to an IRS in $A \rtimes \Gamma$, prove two facts about finite measure preserving linear actions in $\S 2.2$, and prove the result about transverse IRSs in $\S 2.3$. Section 3 concerns IRSs of parabolic subgroups, and in $\S 4$ we prove Theorems 1.1 and 1.2.

\section{IRSs in general semidirect products}

In this section we study semidirect products $G=A \rtimes \Gamma$, where $\Gamma$ acts on $A$ by automorphisms. As above, pr is the natural projection $G \rightarrow \Gamma$.

\subsection{The cocycle $S_{H}$}

Let $H$ be a subgroup of $G$. For each $M \in \operatorname{pr} H$ let

$$
S_{H}(M)=\{v \in A:(v, M) \in H\} .
$$

Then $S_{H}(I)=H \cap A$ is a subgroup of $A$ where $I \in \Gamma$ denotes the identity element.

Let $(v, M),(w, N) \in H$. Then $(v, M)(w, N)=(v \cdot M w, M N) \in H$. It follows that

$$
S_{H}(M N)=S_{H}(M) \cdot M S_{H}(N),
$$

where multiplication here denotes that of sets: $B \cdot C=\{b \cdot c: b \in B, c \in C\}$.

Claim 2.1. The image of $S_{H}$ is in $A / S_{H}(I)$, and $S_{H}: \operatorname{pr} H \rightarrow A / S_{H}(I)$ is a cocycle.

Proof. By (2), it suffices to show that the image of $S_{H}$ is in $A / S_{H}(I)$.

If we substitute $M=I$ into (2) then we get

$$
S_{H}(N)=S_{H}(I) \cdot S_{H}(N),
$$

and so each $S_{H}(N)$ must be a union of cosets of the group $S_{H}(I)$. Substituting $N=M^{-1}$ into (2) yields

$$
S_{H}(I)=S_{H}(M) \cdot M S_{H}\left(M^{-1}\right) .
$$

Hence $S_{H}(M)$ and $M S_{H}\left(M^{-1}\right)$ are cosets of $S_{H}(I)$ in $A$ - i.e. elements of $A / S_{H}(I)$ - with

$$
S_{H}(M)^{-1}=M S_{H}\left(M^{-1}\right) .
$$

We end this section with a useful observation. As we will apply it only when $A$ is abelian, we use additive notation here. Let $(w, N)$ be an arbitrary element of $G$, and let $(v, M) \in H$. Then $(v, M)^{(w, N)}=\left(N^{-1} v+N^{-1}(M-I) w, M^{N}\right) \in H^{(w, N)}$. (Here, $a^{b}=b^{-1} a b$.) Hence

$$
S_{H^{(w, N)}}\left(M^{N}\right)=N^{-1} S_{H}(M)+N^{-1}(M-I) w .
$$




\subsection{Group actions preserving finite measures}

Here are three brief lemmas we will need in the next section.

Lemma 2.2. Suppose a group $Z$ acts linearly on $\mathbb{R}^{d}$ preserving a finite measure $m$, and $V=\operatorname{Span}(\operatorname{supp} m)$. Then the image of the map $Z \longrightarrow \mathrm{GL}(V)$ is precompact.

The proof is similar to an argument of Furstenberg used in his proof of the Borel density theorem [11, Lemma 3].

Proof. Restricting, it suffices to prove the lemma when $\operatorname{Span}(\operatorname{supp} m)=\mathbb{R}^{d}$. Let $\left(z_{n}\right)$ be a sequence in $Z$. After passing to a subsequence, we can assume that there is some subspace $W \subset \mathbb{R}^{d}$ such that the maps $\left.z_{n}\right|_{W}$ converge to some linear map $z: W \longrightarrow \mathbb{R}^{d}$, while $z_{n}(x) \rightarrow \infty$ if $x \in \mathbb{R}^{d} \backslash W$. For instance, one can take $W$ to be any subspace that is maximal among those for which there exists a subsequence $\left(z_{n_{k}}\right)$ with the property that $z_{n_{k}}(x)$ is bounded for all $x \in W$, and then pass to a subsequence of such a subsequence.

If in the above, we always have $W=\mathbb{R}^{d}$, we are done. So, assume $W \neq \mathbb{R}^{d}$. Pick a metric inducing the one-point compactification topology on $\mathbb{R}^{d} \cup \infty$ and let $D: \mathbb{R}^{d} \cup \infty \longrightarrow \mathbb{R}$ be the distance to the closed set $z(W) \cup \infty$. By the dominated convergence theorem,

$$
\int D(x) d m(x)=\int D\left(z_{n}(x)\right) d m(x) \longrightarrow 0,
$$

so $m$ is supported on $z(W)$. But as $W$ is a proper subspace, so is $z(W)$. This contradicts our assumption that $\operatorname{Span}(\operatorname{supp} m)=\mathbb{R}^{d}$.

Lemma 2.3. Suppose that $G$ is a locally compact second countable group, and the induced action of $Z \leq \operatorname{Aut}(G)$ on the space $\operatorname{Sub}_{G}$ preserves a finite measure $\mu$ that is supported on lattices. Then $Z$ preserves the Haar measure of $G$.

Proof. For some $n$, the set $\mathcal{S}$ of lattices with covolume in $\left[\frac{1}{n}, n\right]$ has positive measure. If $Z$ does not preserve Haar measure $\nu$, there is some $A \in Z$ with $A_{*} \nu=c \nu$ with $c>n^{2}$. The sets $A^{i} \mathcal{S}$, where $i \in \mathbb{Z}$, are then all disjoint and have the same positive measure. This is a contradiction.

Lemma 2.4. Suppose that $\mathbb{R}^{d}=\oplus_{i} \mathcal{L}_{i}$, a direct sum of subspaces, and that $\mu$ is a finite Borel measure on the Grassmannian of $k$-dimensional subspaces of $\mathbb{R}^{d}$. Suppose that for each $j$, there is a linear map $A_{j}: \mathbb{R}^{d} \longrightarrow \mathbb{R}^{d}$ that acts as a scalar map $v \mapsto \lambda_{i} v$ on each subspace $\mathcal{L}_{i}$, satisfies $\lambda_{j}>\lambda_{i}$ for $i \neq j$, and induces a map on the Grassmannian that preserves $\mu$. Then $\mu$ is concentrated on subspaces that are direct sums of the $\mathcal{L}_{i}$.

Proof. The argument is similar to that of Lemma 2.2. Under iteration by $A_{j}$, every $k$ dimensional subspace $P$ of $\mathbb{R}^{d}$ converges to a subspace of the form $\mathcal{L}_{j} \oplus P^{\prime}$, where $P^{\prime} \subset \oplus_{i \neq j} \mathcal{L}_{i}$. Applying the dominated convergence theorem, it follows that $\mu$ is concentrated on such subspaces. This works for all $j$, so the lemma follows. 


\subsection{Transverse IRSs}

Let $A$ and $\Gamma$ be locally compact, second countable topological groups, and suppose $\Gamma$ acts by continuous automorphisms on $A$. Let $\Gamma_{\text {triv }}$ be the kernel of the action, and let $G=A \rtimes \Gamma$ be the associated semidirect product.

We call a subgroup $H \leq G$ transverse if $H \cap A=\{0\}$. For example, in the direct product $A \times A$, the diagonal subgroup is transverse, as is the second factor.

Theorem 2.5 (Structure of transverse IRSs in semidirect products, part 1). Suppose $G=$ $\mathbb{R}^{d} \rtimes \Gamma$ and $H$ is a transverse $I R S$ of $G=\mathbb{R}^{d} \rtimes \Gamma$. Then pr $H \leq \Gamma_{\text {triv }}$ almost surely.

Remark 1. Theorem 2.5 also applies when $G=S \rtimes \Gamma$ and $S$ is a closed subgroup of $\mathbb{R}^{d}$. Indeed, the $\Gamma$-action on such an $S$ extends to the span of $S$ to which Theorem 2.5 applies, and any transverse IRS of $G=S \rtimes \Gamma$ induces a transverse IRS of $G=\operatorname{span}(\mathrm{S}) \rtimes \Gamma$.

Remark 2. If the action $\Gamma \circlearrowleft A$ is faithful (as it is, for example, in the case of the special affine groups), then Theorem 2.5 implies there are no nontrivial transverse IRSs of $G$. Also, note that the theorem fails when $A$ is not torsion-free abelian. For instance, if $A$ is finite then a random conjugate of $\Gamma$ is an IRS of $A \rtimes \Gamma$. And if $A$ is not abelian, the antidiagonal

$$
\left\{\left(g, g^{-1}\right) \mid g \in A\right\} \subset A \rtimes A,
$$

where $a \in A$ acts on $x \in A$ by $a(x)=a^{-1} x a$, is a normal subgroup of $A \rtimes A$ that does not project into $A_{\text {triv }}=Z(A)$. However, we expect that for general $A$, if $H$ is a transverse IRS of $A \rtimes \Gamma$, then the action of any element of pr $H$ on $A$ is well-approximated by inner automorphisms of $A$ in some sense.

Proof of Theorem 2.5. Let $H$ be a nontrivial transverse IRS of $G$. In order to get a contradiction, suppose that it is not the case that pr $H \leq \Gamma_{\text {triv }}$ almost surely. Then there is an open subset $U \subset \Gamma$ with compact closure such that $U \cap \Gamma_{\text {triv }}=\emptyset$, and $\operatorname{pr} H \cap U \neq \emptyset$ with positive probability. In addition we choose $U$ small enough so that for some $w \in \mathbb{R}^{d}$, some $0<b_{1}<b_{2} \in \mathbb{R}_{+}$and some linear $L: \mathbb{R}^{d} \longrightarrow \mathbb{R}$, we have that

$$
b_{1} \leq L((M-I) w) \leq b_{2} \text {, for all } M \in U \text {. }
$$

Choose a left Haar measure $\mu_{H}$ on pr $H$. By [5, Claim A.2], this can be done so that the $\mu_{H}$ vary continuously with $H \in \mathrm{Sub}_{G}$, when regarded as measures on $\Gamma \geq \operatorname{pr} H$.

Because $H$ is transverse, $S_{H}(M)$ is a single element of $\mathbb{R}^{d}$ for any $M \in \operatorname{pr} H$. Selecting first a random $H \in \operatorname{Sub}_{G}$ with pr $H \cap U \neq \emptyset$, and then a $\mu_{H}$-random $M \in \operatorname{pr} H \cap U$, we can interpret the cocycle $S_{H}(M)$ as a $\mathbb{R}^{d}$-valued random variable. Here, note that $\mu_{H}(\operatorname{pr} H \cap U)$ is always finite and nonzero, since pr $H \cap U$ is nonempty, pre-compact and open in $H$.

Taking $w \in \mathbb{R}^{d}$ as in the first paragraph of the proof, let $H^{w}=(w, I)^{-1} H(w, I)$. Since pr $H=\operatorname{pr} H^{w}$, we get a map $(H, M) \mapsto\left(H^{w}, M\right)$ defined on the domain

$$
\left\{(H, M) \mid H \in \mathrm{Sub}_{G}, \operatorname{pr} H \cap U \neq \emptyset, M \in \operatorname{pr} H \cap U\right\}
$$


of the random variable $S_{H}(M)$. As $H$ is an IRS, this map is measure preserving, so the distributions of $S_{H^{w}}(M)$ and $S_{H}(M)$ are equal, say to a probability measure $m_{U}$ on $\mathbb{R}^{d}$.

By (3), we have $S_{H^{w}}(M)=S_{H}(M)+(M-I) w$ for all $M \in \operatorname{pr} H=\operatorname{pr} H^{w}$. Iterating the conjugation by $w$ and using (4),

$$
L\left(S_{H}(M)\right)+n b_{1} \leq L\left(S_{H^{n w}}(M)\right) \leq L\left(S_{H}(M)\right)+n b_{2}, \forall n \in \mathbb{N} .
$$

This contradicts the fact that $m_{U}$ is a probability measure. For suppose $\left[a_{1}, a_{2}\right] \subset \mathbb{R}$ is an interval with $m_{U}\left(L^{-1}\left(\left[a_{1}, a_{2}\right]\right)\right)>0$. For a sufficiently sparse sequence $n_{k} \in \mathbb{N}$, the intervals $\left[a_{1}+n_{k} b_{1}, a_{2}+n_{k} b_{2}\right] \subset \mathbb{R}$ are all disjoint. Hence,

$$
1 \geq \sum_{k} m_{U}\left(L^{-1}\left[a_{1}+n_{k} b_{1}, a_{2}+n_{k} b_{2}\right]\right) \geq \sum_{k} m_{U}\left(L^{-1}\left[a_{1}, a_{2}\right]\right)=\infty .
$$

This contradiction proves the theorem.

Theorem 2.6 (Structure of transverse IRSs in semidirect products, part 2). Suppose $G=$ $A \rtimes \Gamma, A$ is a simply connected nilpotent Lie group, $H$ is a transverse IRS of $G=A \rtimes \Gamma$ and $\lambda$ is the law of $H$. Let

$$
\mathcal{H}=\cup_{H \in \operatorname{supp} \lambda} H .
$$

If $\mathcal{V} \subseteq A$ is the Zariski closure of the set of first coordinates of all $(v, M) \in \mathcal{H}$, then $\mathcal{V}$ is $\Gamma$-invariant and the image of the map $Z(\operatorname{pr} \mathcal{H}) \longrightarrow \operatorname{Aut}(\mathcal{V})$ is precompact.

Here $Z(\operatorname{pr} \mathcal{H})$ denotes the centralizer of $\operatorname{pr} \mathcal{H}$ in $\Gamma$, and the Zariski closure of a subset of $A$ is the smallest connected Lie subgroup of $A$ containing that subset.

Remark 3. Theorem 2.6 also applies when $G=S \rtimes \Gamma$ and $S$ is a closed subgroup of some simply connected nilpotent Lie group $A$. Indeed, the $\Gamma$-action on such an $S$ extends to the Zariski closure $\bar{S}$ [15, Theorem 2.11], to which Theorem 2.6 applies, and any transverse IRS of $G=S \rtimes \Gamma$ induces a transverse IRS of $G=\bar{S} \rtimes \Gamma$. See [15, Chapter II] for more information about the 'Zariski closure' operation in simply connected nilpotent Lie groups, which behaves very similarly to 'span' in $\mathbb{R}^{d}$.

Remark 4. To illustrate Theorem 2.6, suppose $A=\Gamma=\mathbb{R}^{2}$ and $(s, t) \in \Gamma$ acts by a rotation on $A$ with angle $s$. Then if

$$
H_{\theta}=\{((t \cos \theta, t \sin \theta),(0, t)) \mid t \in \mathbb{R}\} \leq A \rtimes \Gamma,
$$

we obtain a transverse IRS of $G=A \rtimes \Gamma$ by randomly picking $\theta \in[0,2 \pi]$ against Lebesgue measure. Here, the centralizer $Z(\operatorname{pr} \mathcal{H})$ is all of $\Gamma$, which acts compactly on $A$.

Proof of Theorem 2.6. The $\Gamma$-invariance of $\mathcal{V}$ is immediate. For if $N \in \Gamma$ and $(v, M) \in \mathcal{H}$,

$$
(e, N)^{-1}(v, M)(e, N)=\left(N^{-1} v, N^{-1} M N\right)
$$


Here, we write $e$ for the identity element since $A$ is not necessarily abelian. As $\operatorname{supp} \lambda$ is conjugation invariant, the set of all $v \in A$ such that $(v, M) \in \mathcal{H}$ for some $M$ is $\Gamma$-invariant. Hence, its Zariski closure $\mathcal{V}$ is also $\Gamma$-invariant.

As in the proof of Theorem 2.5, choose $U \subset \Gamma$ with compact closure such that pr $H \cap U \neq \emptyset$ with positive probability. Let $N \in Z(\operatorname{pr} \mathcal{H})$ and write $H^{N}=(e, N)^{-1} H(e, N)$. Substituting $N^{-1} M N=M$ in (7) we see that pr $H=\operatorname{pr} H^{N}$, so as before the distribution of $S_{H^{N}}(M)$ is the same as $m_{U}$, the distribution of $S_{H}(M)$. Now, though, (7) implies that

$$
S_{H^{N}}(M)=N^{-1}\left(S_{H}(M)\right)
$$

So, the measure $m_{U}$ on $A$ is $Z(\operatorname{pr} \mathcal{H})$-invariant.

Since $A$ is a simply connected nilpotent Lie group, there is a diffeomorphism $\log : A \longrightarrow \mathfrak{a}$ to the Lie algebra $\mathfrak{a}$ that is an inverse for the Lie group exponential map [13, 1.127]. Then $\log _{*} m_{U}$ is a probability measure on $\mathfrak{a}$ that is invariant under the induced action of $Z(\operatorname{pr} \mathcal{H})$ on $\mathfrak{a}$. By Lemma 2.2, $Z(\operatorname{pr} \mathcal{H})$ acts precompactly on the span $V_{U}=\operatorname{Span}\left(\operatorname{supp} \log _{*} m_{U}\right)$, and therefore it acts precompactly on the sum $V$ of all $V_{U}$, as $U$ ranges over all possible choices. But the Zariski closure $\mathcal{V}=\exp (V)$, so then $Z(\operatorname{pr} \mathcal{H})$ acts precompactly on $\mathcal{V}$ as well.

We present an easy corollary of Theorem 2.5:

Corollary 2.7. The only ergodic IRSs of the affine group $\mathbb{R} \rtimes \mathbb{R}^{+}$are the point masses on its closed, normal subgroups: $\{e\}, \mathbb{R}, \mathbb{R} \rtimes \mathbb{R}^{+}$and $\mathbb{R} \rtimes\left\{\alpha^{n} \mid n \in \mathbb{Z}\right\}$, where $\alpha>0$.

Note that this stands in contrast to other metabelian groups (e.g., lamplighter groups) that have a rich set of invariant random subgroups [8].

Proof of Corollary 2.7. Let $H$ be a non-trivial ergodic IRS of $\mathbb{R} \rtimes \mathbb{R}^{+}$. If $H$ is transverse, then pr $H=\{1\} \in \mathbb{R}_{+}$, by Theorem 2.5. Hence $H=\{e\}$.

Otherwise, the random subgroup $H \cap \mathbb{R} \subset \mathbb{R}$ is nontrivial almost surely, and its law is invariant under the $\mathbb{R}^{+}$action (i.e., multiplication by a scalar). So, $H \cap \mathbb{R}=\mathbb{R}$ almost surely, and $H=\mathbb{R} \rtimes$ pr $H$. But pr $H$ is an ergodic IRS of $\mathbb{R}^{+}$, and thus must be a point mass on either $\{1\}, \mathbb{R}^{+}$or $\mathbb{R} \rtimes\left\{\alpha^{n} \mid n \in \mathbb{Z}\right\}$, where $\alpha>0$. We have thus proved the claim.

\section{$3 \quad$ IRSs of parabolic subgroups}

To recap our notation: $W=S_{1} \oplus \cdots \oplus S_{n}$ is a real vector space, $\mathcal{F}$ is the associated flag

$$
0=W_{0}<W_{1}<\cdots<W_{n}=W, \quad W_{k}=\oplus_{i=1}^{k} S_{i}
$$

$P<\mathrm{SL}(W)$ is the parabolic subgroup stabilizing $\mathcal{F}, V<P$ is the unipotent subgroup of all $A \in P$ that act trivially on each of the factors $W_{i} / W_{i-1}$, and

$$
P=V \rtimes R, \quad R=\left\{\left(A_{1}, \ldots, A_{n}\right) \in \prod_{i=1}^{n} \mathrm{GL}\left(S_{i}\right) \mid \prod_{i} \operatorname{det} A_{i}=1\right\}
$$


Also, $\mathcal{E} \subset\{1, \ldots, n\}^{2}$ will denote a subset of pairs $(i, j)$ with $i<j$ that is closed under 'going up' and 'going to the right', and we will let $V_{\varepsilon}<P$ be the normal subgroup consisting of all matrices that are equal to the identity matrix except at entries corresponding to elements of $\mathcal{E}$. Let $\mathcal{K}_{\varepsilon}<R$ be the kernel of the $R$-action (by conjugation) on $V / V_{\mathcal{E}}$.

The goal of this section is to prove Theorem 1.3, i.e. that the ergodic IRSs of $P$ are exactly the random subgroups of the form $V_{\varepsilon} \rtimes K$, where $K$ is an ergodic IRS of $\mathcal{K}_{\varepsilon}$.

We start with the following lemma.

Lemma 3.1. Suppose that $H$ is an invariant random subgroup of $P$ that lies in $V$. Then almost surely, $H=V_{\varepsilon}$ for some $\mathcal{E}$.

Proof. Regard $V$ as the space of upper unitriangular block matrices, where the $i j^{\text {th }}$ entries is in $\mathcal{L}\left(S_{i}, S_{j}\right)$. It suffices to show that almost surely, $H$ is a 'matrix entry subgroup', i.e. a subgroup determined by prescribing that some fixed subset of the matrix entries are all zero. As there are only finitely many such subgroups, it will follow that almost surely, $H$ is a matrix entry subgroup of $V$ that is a normal subgroup of $P$. A quick computation with elementary matrices shows that the only such subgroups are the $V_{\varepsilon}$ described above.

Let $H_{0}$ and $\bar{H}$ be the identity component and Zariski closure of $H$, respectively, recalling that the Zariski closure of a subgroup is the smallest connected Lie subgroup of $V$ containing it. (See [15, Chapter II].) Then $H_{0}$ and $\bar{H}$ are both $R$-invariant random subgroups of $V$.

Let $\mathfrak{h}_{0}$ and $\overline{\mathfrak{h}}$ be the associated Lie algebras, which are $R$-invariant random subspaces of the Lie algebra $\mathfrak{v}$ of $V$. One can identify $\mathfrak{v}$ with the set of all strictly upper triangular block matrices, where the $i j^{\text {th }}$ entry is an element of $\mathcal{L}\left(S_{i}, S_{j}\right)$. If we identify $\mathcal{L}\left(S_{i}, S_{j}\right)$ with the subspace of $\mathfrak{v}$ consisting of matrices that are nonzero at most in the $i j^{\text {th }}$ entry, then

$$
\mathfrak{v}=\oplus_{i<j} \mathcal{L}\left(S_{i}, S_{j}\right)
$$

The action $R \circlearrowright \mathfrak{v}$ leaves all the factors $\mathcal{L}\left(S_{i}, S_{j}\right)$ invariant. Moreover, if $k<l$ the matrix in $R$ that has a $2 I$ in the $k k^{t h}$ entry and a $\frac{1}{2} I$ in the $l l^{t h}$ entry (and is otherwise equal to the identity matrix) acts as a scalar matrix $\lambda I$ on each $\mathcal{L}\left(S_{i}, S_{j}\right)$, where

$$
\lambda= \begin{cases}4 & (i, j)=(k, l) \\ 2 & i=k, j \neq l \text { or } j=l, i \neq k \\ \frac{1}{2} & i=l \text { or } j=k, \text { and } i \neq j \\ 1 & \text { otherwise }\end{cases}
$$

So, by Lemma 2.4, almost surely both $\mathfrak{h}_{0}$ and $\overline{\mathfrak{h}}$ are direct sums of some of the factors $\mathcal{L}\left(S_{i}, S_{j}\right)$, i.e. the groups $H_{0}$ and $\bar{H}$ are matrix entry subgroups. If they are the same, we are done since then $H=H_{0}=\bar{H}$ is a matrix entry subgroup.

Passing to a positive measure $R$-invariant subset, we may thus assume that almost surely $H_{0}$ and $\bar{H}$ are fixed matrix entry subgroups and that $H_{0} \subsetneq H$. As $H$ is an IRS of $P$, $H_{0}$ is a normal subgroup of $P$. We can then project $H$ to a $P$-invariant random lattice of the quotient group $\bar{H} / H_{0}$. Lemma 2.3 implies that the $P$ action on $\bar{H} / H_{0}$ preserves Haar 
measure. But if $\mathcal{D}$ is the set of matrix entries that are free to take on any value in $\bar{H}$ and prescribed to be zero in $H_{0}$, there is a diffeomorphism

$$
\bar{H} / H_{0} \longrightarrow \oplus_{(i, j) \in \mathcal{D}} \mathcal{L}\left(S_{i}, S_{j}\right)
$$

that takes a matrix in $\bar{H}$ to the list of its $\mathcal{D}$-entries. If Lebesgue measures are chosen on the Euclidean spaces $\mathcal{L}\left(S_{i}, S_{j}\right)$, the resulting product measure pulls back to a Haar measure on $\bar{H} / H_{0}$. So, one can witness that the action $R \circlearrowright \bar{H} / H_{0}$ does not preserve Haar measure as follows. Let $i_{\text {min }}$ be the minimum $i$ such that there is some $(i, j) \in \mathcal{D}$, and $i_{\max }$ be the maximum $i$ such that there is some $(j, i) \in \mathcal{D}$, and define $A \in R$ by letting

$$
A_{i i}= \begin{cases}2 I & i=i_{\min } \\ \frac{1}{2} I & i=i_{\max } \\ I & \text { otherwise }\end{cases}
$$

Then there are no entries of $\mathcal{D}$ directly above the $i_{\text {min }}$ diagonal entry, and no entries to the right of the $i_{\text {max }}$ diagonal entry. Hence, all eigenvalues of the (linear) action of $A$ on $\oplus_{(i, j) \in \mathcal{D}} \mathcal{L}\left(S_{i}, S_{j}\right)$ are equal to either 1 or 2 , so $A$ cannot preserve Lebesgue measure.

Now suppose that $H$ is an ergodic IRS of $P=V \rtimes R$. Lemma 3.1 implies that there is some $\mathcal{E}$ such that $H \cap V=V_{\varepsilon}$ almost surely. Applying Theorem 2.5 to the transverse IRS that is the projection of $H$ to $\left(V / V_{\varepsilon}\right)^{a b} \rtimes R$, where $(\cdot)^{a b}$ is abelianization, we see that pr $H \subset R$ almost surely acts trivially on $\left(V / V_{\varepsilon}\right)^{a b}$. But if $\mathcal{A}$ is the set of super diagonal entries in our block matrices that do not lie in $\mathcal{E}$, there is an isomorphism

$$
\left(V / V_{\varepsilon}\right)^{a b} \longrightarrow \oplus_{(i, j) \in \mathcal{A}} \mathcal{L}\left(S_{i}, S_{j}\right)
$$

that comes from taking a matrix in $V$ to its list of $\mathcal{A}$-entries. It follows that a matrix in $R$ acts trivially on $\left(V / V_{\varepsilon}\right)^{a b}$ if and only if it acts trivially on $V / V_{\varepsilon}$ : triviality of the $\left(V / V_{\varepsilon}\right)^{a b}$-action is enough to force the conditions on diagonal entries indicated in the matrix (1) from the introduction. Hence, pr $H$ almost surely lies in the kernel $\mathcal{K}_{\varepsilon}$ of the $V / V_{\varepsilon}$-action as desired.

We now know that $H \cap V=V_{\varepsilon}$ and pr $H \subset \mathcal{K}_{\varepsilon}$ almost surely. We would like to conclude that $H$ has the form $V_{\varepsilon} \rtimes K$ for some IRS $K<K_{\varepsilon}$. Note that this is not immediately obvious - the diagonal in $\mathbb{R}^{2}$ is a normal subgroup that intersects the first factor trivially, but does not split as a product of subgroups of the two factors. By Theorem 2.6, we know that the centralizer $Z(\operatorname{pr} H) \subset R$ acts precompactly on $X \subset V / V_{\mathcal{E}}$, where $\mathcal{X}$ is the Zariski closure in $V / V_{\varepsilon}$ of the projections of all first coordinates of elements $(v, M) \in H$. If $\mathcal{X}=\left\{V_{\varepsilon}\right\}$, we are done, since then the first coordinates of all $(v, M) \in H$ lie in $V_{\varepsilon}=H \cap V$ and $H$ must have the form $V_{\varepsilon} \rtimes K$ for some IRS $K<\mathcal{K}_{\varepsilon}$.

So, we may assume that $X \mathcal{V}_{\mathcal{E}} \supsetneq V_{\mathcal{E}}$. Picking a matrix $B$ in the difference, there is some entry $(i, j) \notin \mathcal{E}$ in which $B$ is nonzero. The centralizer $Z(\operatorname{pr} H)$ contains all elements of $R$ all of whose diagonal entries are scalars, so in particular it contains the matrix whose eigenvalues $\lambda$ are listed in (8) above. The action of this matrix on $B$ scales the $(i, j)$ entry by 4 , so $Z(\operatorname{pr} H)$ does not act pre-compactly on $X$, and we have a contradiction. 


\section{IRSs of special affine groups}

Using Theorems 2.5 and 2.6, it is now fairly easy to prove the results on IRSs of special affine groups stated in the introduction.

Proof of Theorem 1.1. Let $H$ be a nontrivial ergodic IRS of $\mathbb{R}^{d} \rtimes \mathrm{SL}_{d}(\mathbb{R})$. Suppose that $H \cap \mathbb{R}^{d}=\{0\}$ almost surely. As the action $\mathrm{SL}_{d}(\mathbb{R}) \circlearrowright \mathbb{R}^{d}$ is faithful, Theorem 2.5 implies that $H$ is trivial. So, $H \cap \mathbb{R}^{d}$ is almost surely some nontrivial subgroup of $\mathbb{R}^{d}$.

In order to prove $H \cap \mathbb{R}^{d}$ is either a lattice or $\mathbb{R}^{d}$, it suffices to prove that the Zariski closure of $H \cap \mathbb{R}^{d}$ is almost surely $\mathbb{R}^{d}$. If not, we get for some $1 \leq k \leq d-1$, a $\mathrm{SL}_{d}(\mathbb{R})$ invariant probability measure on the Grassmannian of $k$-dimensional subspaces of $\mathbb{R}^{d}$. In the terminology of Furstenberg [11], $\mathrm{SL}_{d}(\mathbb{R})$ is a m.a.p. group, so this measure must be concentrated on $\mathrm{SL}_{d}(\mathbb{R})$-invariant points. (Apply [11, Lemma 3] to the $k^{\text {th }}$ exterior power of $\mathbb{R}^{d}$.) However, no nontrivial subspaces of $\mathbb{R}^{d}$ are $\mathrm{SL}_{d}(\mathbb{R})$-invariant.

Now suppose $H \cap \mathbb{R}^{d}$ is a lattice (almost surely). Let $\mu$ denote the law of $H$. By decomposing $\mu$ over the map $H \mapsto H \cap \mathbb{R}^{d}$, we can write $\mu=\int \mu_{\Lambda} d \nu(\Lambda)$ where $\nu$ is the pushforward of $\mu$ under $H \mapsto H \cap \mathbb{R}^{d}$ and $\mu_{\Lambda}$ is concentrated on the set of subgroups $H$ such that $H \cap \mathbb{R}^{d}=\Lambda$. By ergodicity $\nu$ is supported on the set of lattices of some fixed covolume $c>0$. Moreover $\nu$ is $\mathrm{SL}_{d}(\mathbb{R})$ invariant since the map $H \mapsto H \cap \mathbb{R}^{d}$ is equivariant. Since $\mathrm{SL}_{d}(\mathbb{R})$ acts transitively on this set of lattices, it follows that $\nu$ must be the Haar measure.

By equivariance, we must have $\mu_{g \Lambda}=g_{*} \mu_{\Lambda}$ for $g \in \mathrm{SL}_{d}(\mathbb{R})$ and $\nu$-a.e. $\Lambda$. Because $\mathrm{SL}_{d}(\mathbb{R})$ acts transitively on the set of lattices with fixed covolume, we can assume without loss of generality that $\mu_{g \Lambda}=g_{*} \mu_{\Lambda}$ holds for every $g \in \mathrm{SL}_{d}(\mathbb{R})$ and lattice $\Lambda$.

We claim that $\mu_{\Lambda}$-a.e. $H$ is contained in $\Lambda \rtimes \mathrm{SL}(\Lambda)$. First let $(v, M) \in H$. For any $w \in \Lambda$ we have that $(w, I) \in H$, and so

$$
(v, M)(w, I)(v, M)^{-1}=(M w, I) \in H \cap \mathbb{R}^{d}=\Lambda .
$$

Because $w \in \Lambda$ is arbitrary, $M \in \mathrm{SL}(\Lambda)$. Next observe that the law of $H$ is invariant under conjugation by $\Lambda \rtimes \mathrm{SL}(\Lambda)$. So if there exists $M \in \mathrm{SL}(\Lambda)$ such that $S_{H}(M) \neq \Lambda$ with positive probability then $M H M^{-1} \cap \mathbb{R}^{d} \neq \Lambda$ with positive probability. This contradiction shows that $S_{H}(M)=\Lambda$ almost surely which implies $H \leq \Lambda \rtimes \mathrm{SL}(\Lambda)$. Thus $\mu_{\Lambda}$ is the law of an IRS of $\Lambda \rtimes \mathrm{SL}(\Lambda)$. This IRS must be ergodic because $\mu$ is ergodic.

Proof of Theorem 1.2. Let $H$ be a non-trivial, ergodic IRS of $G=\mathbb{Z}^{d} \rtimes \mathrm{SL}_{d}(\mathbb{Z})$. Then $H \cap \mathbb{Z}^{d}$ is a random subgroup of $\mathbb{Z}^{d}$ whose law is invariant to the $\mathrm{SL}_{d}(\mathbb{Z})$ action. Note that since the action $\mathrm{SL}_{d}(\mathbb{Z}) \circlearrowright \mathbb{Z}^{d}$ is faithful, Theorem 2.5 implies that $H \cap \mathbb{Z}^{d} \neq\{0\}$. Since there are only countably many subgroups of $\mathbb{Z}^{d}$, the distribution of $H \cap \mathbb{Z}^{d}$ must be concentrated on a single, finite $\mathrm{SL}_{d}(\mathbb{Z})$-orbit. So, $H \cap \mathbb{Z}^{d}$ is almost surely finite index in $\mathbb{Z}^{d}$.

Let $O=\left\{M\left(H \cap \mathbb{Z}^{d}\right): M \in \mathrm{SL}_{d}(\mathbb{Z})\right\}$ be the orbit of $H \cap \mathbb{Z}^{d}$ under the $\mathrm{SL}_{d}(\mathbb{Z})$ action. Now, the intersection of the groups in this orbit is also finite index in $\mathbb{Z}^{d}$, and is furthermore $\mathrm{SL}_{d}(\mathbb{Z})$-invariant, and so must equal $n \mathbb{Z}^{d}$ for some $n \in \mathbb{N}$.

Recall that $G_{n}=\left(n \mathbb{Z}^{d}\right) \rtimes \Gamma(n)$, and let $H_{n}=H \cap G_{n}$, a finite index subgroup of $H$. Using the cocycle notation of $\S 2.1$, for any $M \in \operatorname{pr} H_{n}$ it holds that $S_{H}(M)=S_{H}(I):=H \cap \mathbb{Z}^{d}$, since 
otherwise $S_{H}(M)$ is a non-trivial coset of $S_{H}(I)$, and its intersection with $n \mathbb{Z}^{d}$, a subgroup of $S_{H}(I)$, is trivial, thus excluding $M$ from pr $H_{n}$. It follows that $H_{n}=\left(n \mathbb{Z}^{d}\right) \rtimes\left(\operatorname{pr} H_{n}\right)$. This completes the proof of Theorem 1.2.

\section{References}

[1] M. Abert, N. Bergeron, I. Biringer, T. Gelander, N. Nikolov, J. Raimbault, and I. Samet. On the growth of $L^{2}$-invariants for sequences of lattices in lie groups. arXiv preprint arXiv:1210.2961, 2012.

[2] M. Abert, Y. Glasner, and B. Virag. Kesten's theorem for Invariant Random Subgroups. ArXiv e-prints, Jan. 2012.

[3] U. Bader, B. Duchesne, J. Lécureux, and P. Wesolek. Amenable invariant random subgroups. Israel Journal of Mathematics, pages 1-24, 2014.

[4] R. Benedetti and C. Petronio. Lectures on hyperbolic geometry. Universitext. SpringerVerlag, Berlin, 1992.

[5] I. Biringer and O. Tamuz. Unimodularity of invariant random subgroups. arxiv.org/abs/1402.1042, 2014.

[6] L. Bowen. Invariant random subgroups of the free group. arXiv preprint arXiv:1204.5939, 2012.

[7] L. Bowen. Random walks on random coset spaces with applications to furstenberg entropy. Inventiones Mathematicae, 196(2):485-510, 2014.

[8] L. Bowen, R. Grigorchuk, and R. Kravchenko. Invariant random subgroups of lamplighter groups. Israel Journal of Mathematics, 207(2):763-782, 2015.

[9] C. Chabauty. Limite d'ensembles et géométrie des nombres. Bull. Soc. Math. France, 78:143-151, 1950.

[10] A. Diatta and B. Foreman. Lattices in contact Lie groups and 5-dimensional contact solvmanifolds. arXiv preprint arXiv:0904.3113, 2012.

[11] H. Furstenberg. A note on Borel's density theorem. Proc. Amer. Math. Soc., 55(1):209$212,1976$.

[12] Y. Hartman and O. Tamuz. Furstenberg entropy realizations for virtually free groups and lamplighter groups. Journal d'Analyse Mathmatique, 126(1):227-257, 2015.

[13] A. W. Knapp. Lie groups beyond an introduction, volume 140. Springer Science \& Business Media, 2013. 
[14] R. R. Phelps. Lectures on Choquet's theorem, volume 1757 of Lecture Notes in Mathematics. Springer-Verlag, Berlin, second edition, 2001.

[15] M. S. Raghunathan. Discrete subgroups of Lie groups. Springer-Verlag, New York, 1972. Ergebnisse der Mathematik und ihrer Grenzgebiete, Band 68.

[16] G. Stuck and R. J. Zimmer. Stabilizers for ergodic actions of higher rank semisimple groups. Annals of Mathematics, pages 723-747, 1994.

[17] S. Thomas and R. Tucker-Drob. Invariant random subgroups of strictly diagonal limits of finite symmetric groups. Bulletin of the London Mathematical Society, 46(5):10071020, 2014.

[18] R. D. Tucker-Drob. Weak equivalence and non-classifiability of measure preserving actions. Ergodic Theory and Dynamical Systems, 35(01):293-336, 2015.

[19] A. Vershik. Totally nonfree actions and infinite symmetric group. ArXiv e-prints, Sept. 2011. 